\title{
Diacronie
}

Studi di Storia Contemporanea

$N^{\circ} 7,3 \mid 2011$

«Spagna Anno Zero»: la guerra come soluzione

\section{Prologo: La guerra civile spagnola, settantacinque anni dopo}

\section{Alfonso Botti}

\section{Q OpenEdition}

1 Journals

Edizione digitale

URL: http://journals.openedition.org/diacronie/3143

DOI: 10.4000/diacronie.3143

ISSN: 2038-0925

Editore

Association culturelle Diacronie

Notizia bibliografica digitale

Alfonso Botti, «Prologo: La guerra civile spagnola, settantacinque anni dopo », Diacronie [Online], $\mathrm{N}^{\circ} 7$,

3 | 2011, Messo online il 29 juillet 2011, consultato il 23 septembre 2020. URL : http://

journals.openedition.org/diacronie/3143; DOI : https://doi.org/10.4000/diacronie.3143

Questo documento è stato generato automaticamente il 23 settembre 2020.

Creative Commons License 


\title{
Prologo: La guerra civile spagnola, settantacinque anni dopo
}

\author{
Alfonso Botti
}

1 Nella guerra civile spagnola vennero al pettine secolari contraddizioni interne che le radicali riforme del primo biennio repubblicano non riuscirono a risolvere, ma la posta in gioco per quasi tre anni furono i destini dell'Europa. Poi il Vecchio continente cadde nel baratro da cui riemerse nel 1945, mentre gli spagnoli vennero lasciati soli a pagare le conseguenze della vittoria di Franco e

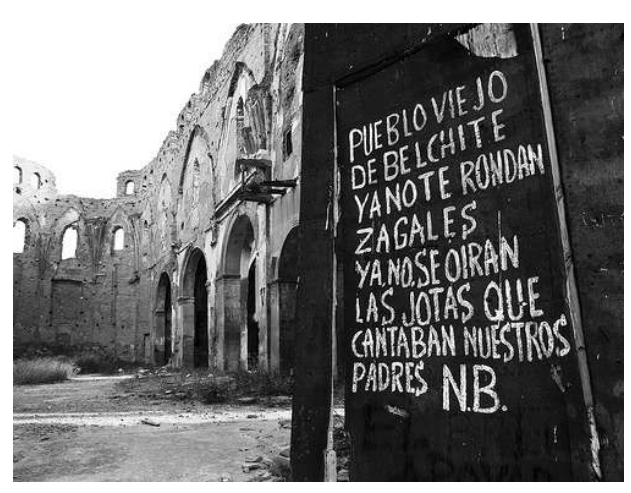
privati della sovranità fino al 1977. I tre quarti di secolo che ci separano dalla sollevazione militare del 17-18 luglio 1936, il cui fallimento scatenò la più emblematica delle guerre civili del Novecento, non hanno attenuato l'interesse per la tragedia del 1936-39 e per la sua scia nella memoria spagnola e non solo europea.

2 Ne sono riprova, accanto ad altri recenti lavori dei quali si dirà più avanti (senza nessuna pretesa di completezza, è bene precisare), i ventiquattro contributi raccolti in questo numero di "Diacronie": ventuno propriamente storiografici, ai quali si aggiungono tre apporti documentali, tra i quali spicca la testimonianza di un combattente nelle Brigate Internazionali austriaco, già edito in lingua spagnola e qui riproposto in italiano. Varie le linee tematiche attorno alle quali essi si raggruppano. Una leggera prevalenza è data al tema della memoria, indagato nei contesti francese, italiano, tedesco, austriaco e internazionale in senso lato. Nel primo caso attraverso quella dei figli dei rifugiati spagnoli (Federica Luzi), nel secondo attraverso quella dell'antifascismo e il profilo del garibaldino toscano Aureliano Santini (Enrico Acciai), nel terzo studiando le rappresentazioni dell'esilio della sinistra tedesca (Claudia Roesch), poi attraverso la già segnalata testimonianza dell'anziano combattente 
austriaco (Gerhard Hoffmann) e, da ultimo, con un'ampia panoramica sulle fonti relative alle Brigate Internazionali (Lourdes Prades-Artigas, Montserrat SebastiáSalat). In parte al tema della memoria è dedicato anche uno dei contributi sull'anarchismo, peraltro attento alla dimensione etica di quella militanza (Carmen González Martín), le cui correnti vengono indagate anche per quanto riguarda il loro ruolo all'interno del campo repubblicano durante il conflitto attraverso un puntuale esame della più recente letteratura (Arnaud Dolidier), mentre della rete di solidarietà anarchica sul piano internazionale si dirà più avanti. Un'altra linea tematica è possibile comporre con gli interventi dedicati alle dinamiche politiche e ad alcuni episodi bellici, sia per quanto concerne quelle parlamentari, con il contributo dedicato alla condotta dei deputati di Castilla-León nei mesi che precedettero il conflitto ( $\mathbf{M}^{\mathrm{a}}$ Concepción Marcos del olmo), sia in merito ai contrasti nell'entroterra valenciano tra socialisti e comunisti, schierati questi ultimi a difesa dei piccoli proprietari agricoli (Sergio Valero Gómez), sia in relazione al ruolo della propaganda, esaminata nel caso di Cartagena, uno dei simboli della resistenza repubblicana (Pedro $\mathbf{M}^{\mathbf{a}}$ Egea Bruno), sia per quanto concerne le tensioni all'interno del fronte ribelle, studiate per il caso di Valladolid, "capitale della sollevazione militare" (Matteo Tomasoni), sia per quanto concerne la battaglia di Guadalajara e l'incontro/scontro tra gli italiani combattenti nei due campi (Valentina Catelan) e sia, infine, in relazioni ai bombardamenti su Barcellona del marzo 1938 (Edoardo Grassia, autore anche di un intervento sull'aviazione legionaria). Né manca la dimensione internazionale, che costituisce l'altra linea attorno alla quale è possibile raccogliere i contributi dedicati alla Solidarietà Internazionale Antifascista d'ispirazione anarchica (Valentin Cionini), il sondaggio effettuato sulla stampa del dipartimento francese della Loire sulle rappresentazioni del conflitto (Pascale Moiron) e i due interventi dedicati all'atteggiamento di Salazar e alla sua politica estera negli anni del conflitto spagnolo (Cécil Goncalvez), accanto al quale si colloca l'analisi delle emissioni radiofoniche di "Rádio Club Português (Alberto Pena Rodríguez), che inducono a soffermare l'attenzione su un aspetto meno noto e meno frequentato dagli storici qual è l'apporto fornito dal dittatore portoghese al successo dei militari ribelli spagnoli. Ultimo, ma non meno interessante, l'asse dedicato agli aspetti culturali che conta sugli apporti dedicati alla difesa del patrimonio artistico da parte della Repubblica (Rebeca Saavedra Arias) e alle tendenze architettoniche della Barcellona repubblicana (Gemma Domènech Casadevall).

3 Complessivamente considerati, si tratta di contributi scientificamente solidi, basati su un considerevole ventaglio di fonti dirette (archivistiche e a stampa) e storiografiche, che offrono una panoramica ampia, che pone uno accanto all'altro sondaggi su aspetti specifici e locali, in alcuni casi indagati per la prima volta, a contributi di carattere più sintetico e generale, senza pretese di completezza ed esaustività (peraltro impossibile per la vastità della materia e della letteratura ad essa dedicata). Una panoramica che vede contributi di studiosi collaudati, accanto a storici in via di formazione e, in alcuni casi, addirittura alle prime armi. Panoramica internazionale non solo perché volta ad alcuni degli aspetti internazionali del conflitto, ma anche per l'apporto di studiosi di diversi paesi europei.

4 Con i pregi, sono da indicare anche alcuni limiti che l'insieme dei contributi, anche in questo caso complessivamente considerati, lasciano intravvedere. In primo luogo quello dell'autoreferenzialità delle storiografie, poco avvezze a tener conto delle ricerche degli altri ispanismi. In secondo luogo la difficoltà (dovuta forse a eccessiva 
prudenza) di misurarsi con i principali nodi interpretativi del conflitto spagnolo, delle sue cause, delle sue dinamiche e del ruolo dei suoi protagonisti collettivi e individuali, attorno ai quali è andata dipanandosi la letteratura degli ultimi decenni fino alla più recente.

La guerra civile fu lotta per la democrazia senza aggettivi contro il fascismo, ultima rivoluzione nell'occidente capitalistico contro la democrazia "borghese" ed epifania dei crimini che Stalin stava perpetrando a Oriente; crociata in difesa della civiltà cristiana $\mathrm{e}$ tra le pagine più nere della Chiesa in età contemporanea; lotta per il diritto di baschi $\mathrm{e}$ catalani a non vedere soffocate la propria identità culturale e aspirazione all'autogoverno; culmine della mobilitazione antifascista internazionale con le Brigate Internazionali e l'impegno degli intellettuali; rivelazione dell'impotenza della ginevrina Società delle Nazioni e vittoria diplomatica del nazi-fascismo, che riuscì a imbrigliare Francia e Gran Bretagna nella farsa del Non-Intervento. Con i bombardamenti sulle città aperte (Madrid, Barcellona e tante altre, oltre al più noto caso di Gernika) fu terreno di sperimentazione per quella guerra totale che avrebbe dato i suoi frutti più nefasti nella Seconda guerra mondiale di cui fu, per certi versi, la prima battaglia. Segnò l'avvio di una dittatura longeva, sanguinaria e illiberale, alla quale la capacità di galleggiamento del suo eroe eponimo garantì la durata, mentre gli equilibri della guerra fredda diedero costante ossigeno. Fu uno scontro tra ideologie totalizzanti, di propaganda, di manipolazioni grossolane (i documenti apocrifi sul presunto colpo di Stato comunista e quelli confezionati dagli agenti stalinisti per "provare" la collusione di Andrés Nin e degli altri dirigenti del POUM con il fascismo, l'attribuzione all'esercito nazionalista basco della distruzione di Gernika, per dire solo delle principali) e fucina in entrambi i campi di miti destinati a durare. All'interno della guerra civile se ne combatterono altre due: quella tra comunisti e socialisti, da una parte, e anarchici e militanti del POUM, dall'altra, e quella che contrappose i cattolici carlisti navarresi ai cattolici nazionalisti baschi. E fu guerra di interpretazioni, che il tempo ha stemperato, almeno in parte, dalle passioni dell'ideologia, lasciandole a interloquire nel luogo deputato del dibattito storiografico, specie dopo l'apertura degli archivi spagnoli dal 1975, di quelli moscoviti dai primi anni Novanta e, dal settembre 2006, dell'Archivio Segreto Vaticano (ASV) per il pontificato di Pio XI.

\section{La violenza politica}

6 Il tema della violenza politica e della repressione nelle retrovie è stato quello più frequentato dagli storici spagnoli (e non solo) dopo il 1975. Il campo fino a quel momento occupato dalle ricostruzioni dei vincitori, ha visto da allora il fiorire di una messe di studi irti di dati raccapriccianti. Ad alimentarli, nell'ultimo decennio, la scoperta di decine di fosse comuni con le spoglie di migliaia di vittime repubblicane, che a sua volta ha animato un movimento rivendicativo civico, la cui spinta ha condotto alla Ley 52/2007, impropriamente chiamata della "memoria storica" ${ }^{1}$ Paul Preston ha da poco compendiato anni di ricerche, sue e di altri studiosi, in un volume che riassume quanto accadde nella Spagna di allora con il termine "olocausto". Violenza prevalentemente spontanea quella dei repubblicani e freddamente pianificata quella dei franchisti, che l'ispanista britannico esamina nei suoi presupposti ideologici e nelle sue applicazioni nelle differenti realtà territoriali in un ponderoso volume, dove tra l'altro sviluppa l'idea, già esposta nella sua biografia di Franco, che i metodi dei militari 
ribelli furono gli stessi impiegati nelle guerre coloniali in Marocco, dalla cui esperienza provenivano in gran parte. Le cifre ancora approssimative e provvisorie che fornisce indicano in 200 mila le donne e gli uomini uccisi nelle retrovie (50 mila circa vittime della repressione repubblicana e le rimanenti di quella franchista), in circa 300 mila i caduti sui fronti di battaglia e in almeno 20 mila i repubblicani fucilati dopo la fine delle ostilità.

\section{Comunismo e ceti medi}

7 Nuove letture sono giunte negli ultimi tempi anche a proposito del Partito comunista spagnolo (PCE). Nota la sua marginalità fino al luglio del '36, era stato Burnett Bolloten, nei primi anni Sessanta ${ }^{3}$, in un'opera che continuò ad arricchire e a riscrivere fino all'edizione postuma del $1989^{4}$, a sostenere che, rafforzatosi con l'aiuto sovietico, il PCE aveva di fatto conquistato il potere insediando alla presidenza del governo un proprio uomo di fiducia con Negrín, mostrando in questo modo quanto ingannevole (il Grand Camouflage del titolo) fosse la propaganda comunista imperniata sulla difesa della democrazia liberale e borghese. Non solo. Bolloten sosteneva che se la Repubblica avesse vinto, la Spagna avrebbe imboccato la via poi percorsa dalle "democrazie popolari" dell'Est europeo. Ammetteva anche che senza sollevazione militare e guerra civile, il comunismo spagnolo sarebbe rimasto minoritario e, quindi, che lungi dallo scongiurare la minaccia sovietica esse avevano fornito a Stalin l'occasione per intervenire nel paese iberico.

8 Un recente studio di Fernando Hernández Sánchez approda, sulla scorta di minuziose ricerche negli archivi del PCE e degli scandagli svolti da altri storici in quelli di Mosca, a un'interpretazione diversa. Più che dalla subdola strategia di conquista del potere e dal ruolo di intermediario degli aiuti sovietici, il PCE accrebbe la propria forza e influenza per la sua capacità di occupare lo spazio politico e sociale lasciato vacante dalla radicalizzazione delle altre forze di sinistra e dallo spostamento su posizioni rivoluzionarie di gran parte del Partito socialista. Uno spazio che riuscì a occupare facendo propria la cultura politica e gli ideali patriottici del progressismo liberale e della tradizione repubblicana riformista di fine Ottocento (giustizia, riforme sociali, progresso, libertà, laicità, istruzione popolare) ${ }^{5}$.

\section{La democrazia e gli altri ceti medi}

9 In definitiva gli ideali di una parte significativa di quei ceti medi che, stando invece all'ultima monografia dall'impianto noltiano (senza però il giustificazionismo dello storico tedesco) di Gabriele Ranzato ${ }^{6}$, dedicata alla grande paura della rivoluzione che s'impossessò di essi dopo la fallita rivoluzione dell'ottobre del 1934 e nei mesi che intercorsero dalla vittoria del Fronte popolare al luglio del 1936, rimasti orfani di rappresentanza politica, finirono tra le braccia della destra eversiva. Ranzato indaga il discorso pubblico della sinistra socialista di Largo Caballero, responsabile assieme ai comunisti di aver favorito la crisi del governo di Fronte Popolare al fine di sostituirlo con un esecutivo che guidasse dall'alto l'avvio dell'agognato processo rivoluzionario. Di qui l'intensificazione degli scioperi, delle aggressioni anticlericali contro chiese e conventi, dei disordini nelle campagne e delle occupazioni delle terre da parte dei contadini, l'escalation della violenza fino all'assassinio di Calvo Sotelo, che rese 
manifesto l'offuscamento dello Stato di diritto e la perdita del monopolio del legittimo utilizzo della violenza. Tra i due schieramenti in via di polarizzazione, un'area sociale e d'opinione (la cosiddetta "Terza Spagna") che non voleva né la rivoluzione comunista, né una dittatura di tipo autoritario o fascista, che rimase stritolata, anche perché rappresentata sul piano politico da uomini poco lungimiranti, troppo deboli o rassegnati, scarsamente democratici e responsabili di una serie di gravi errori: l'aver costretto Alcalá Zamora alle dimissioni; la nomina a capo del governo del debolissimo Casares Quiroga, l'applicazione di una Riforma agraria che, con la clausola dell'“utilità sociale", consentiva espropri della piccola proprietà, gettando nei panico i proprietari; la chiusura delle scuole cattoliche; il trasferimento dei generali Franco e Mola in sedi (rispettivamente le Canarie e la Navarra) che ne agevolarono le trame cospirative; la sottovalutazione dei segnali di imminente golpe militare; la mancata risposta istituzionale all'assassinio di Calvo Sotelo, per dire solo dei principali.

Se Hernández Sánchez mostra la capacità del PCE di rassicurare i ceti medi d'orientamento laico dopo il 18 luglio, Ranzato mette in luce le paure della parte più influenzata dalla Chiesa prima. Non a caso si parla di ceti, una declinazione plurale da articolarsi anche sul piano territoriale, essendo i ceti medi galiziani, per esempio, ben diversi da quelli catalani.

In precedenza Rafael Cruz aveva proposto un'altra lettura degli stessi mesi cruciali, negando il fallimento della Repubblica, ridimensionando il ruolo della violenza politica e l'alterazione dell'ordine pubblico quale principale causa della sollevazione militare ${ }^{7}$. La quale, come ha ricordato più recentemente anche Francisco Alía Miranda, se aveva preso corpo nella forma che sarebbe poi risultata definitiva dall'aprile del 1936, affondava le proprie radici in trame cospirative precedenti, per le quali la paura di una imminente rivoluzione comunista più che un effettivo movente rappresentò un alibi $\mathrm{e}$ uno spauracchio da agitare per preparare il terreno al golpe. Ciò, si badi bene, senza misconoscere il ruolo che ebbe a giocare la paura della rivoluzione, non in uno specifico settore sociale, ma in gran parte della società spagnola e presso le principali potenze straniere ${ }^{8}$.

\section{La Chiesa}

Dati interessanti negli ultimi tempi sono emersi, infine, anche sulla condotta ecclesiastica. La Chiesa spagnola non fu solo vittima ${ }^{9}$, fu anche collusa e complice dei carnefici degli "altri". Non ebbe tra le proprie fila solo martiri, ma anche delatori e militanti fanatici della causa franchista. Si schierò con gli insorti prima che Pio XI prendesse posizione nel discorso di Castel Gandolfo del 14 settembre 1936 e fornì loro le motivazioni ideologiche di cui mancavano per far fronte a una guerra civile imprevista, dopo il fallito golpe. Il cardinale primate, Isidro Gomá, di cui disponiamo ora di tutta la corrispondenza per il periodo della guerra ${ }^{10}$, utilizzò spregiudicatamente i falsi documenti del complotto comunista per giustificare la guerra come "male minore" ${ }^{11}$, assecondò Franco redigendo la Lettera collettiva del luglio del '37 per orientare il cattolicesimo internazionale che non si era lasciato abbagliare dall'idea di "crociata", minimizzò e giustificò sempre le violenze perpetrate dai franchisti. Operò costantemente, insomma, per portare la S. Sede a un più completo sostegno agli insorti, riuscendovi in parte. La nuova documentazione vaticana conferma le diversità di atteggiamento, arricchendo il quadro di nuovi particolari. Sappiamo ora del peso che la 
mancata condanna da parte del governo di Madrid delle violenze anticlericali ebbe nel determinare l'atteggiamento della S. Sede e che prima del discorso del 14 settembre Pio XI aveva pensato di rivolgere un appello per la cessazione delle ostilità, cosa che non fece più in seguito. Abbiamo un quadro più chiaro delle resistenze che il Vaticano frappose a lasciarsi coinvolgere nei tentativi di soluzione negoziata del conflitto proposti dalla diplomazia franco-britannica, ma anche nell'assecondare Franco, che più volte chiese una pubblica condanna dei nazionalisti baschi. Una conferma hanno trovato lo scarso apprezzamento che la S. Sede mostrò per la Lettera collettiva del luglio '37, i timori per l'influenza nazista in terra iberica ${ }^{12}$ e l'impegno profferto per l'“umanizzazione" del conflitto (scambio di prigionieri, sospensione bombardamenti sulle città, commutazione delle pene capitali, ecc.), terreno sul quale $\mathrm{i}$ vertici ecclesiastici romani si mossero quasi sempre in ritardo, a ruota dell'indignata opinione democratica internazionale (anche di parte cattolica). Una riprova che negli ambienti curiali la corrente fredda delle preoccupazioni politico-diplomatiche soffocò il vento caldo della denuncia e della testimonianza della verità.

Sono questi alcuni dei temi attorno ai quali si sta svolgendo da qualche tempo un dibattito storiografico dal quale è lecito attendersi nuove acquisizioni di dettaglio e innovative visioni d'insieme. In questo ambito è da collocare il valido contributo offerto da questo numero di "Diacronie".

\section{NOTE}

1. Il dibattito sui media e in ambito più propriamente storiografico al riguardo è stato assai ampio. I termini essenziali della questione sono ben riassunti in: ÁLVAREZ JUNCO, José, "La ley de víctimas de la Guerra Civil y del Franquismo”, in Historia contemporánea, 2009, n. 38, pp. 145-161; BRUNNER, José, "Ironías de la historia española: Observaciones sobre la política pos-franquista de olvido y de memoria”, ivi, pp. 163-183; ÁLVAREZ JUNCO, José, “Respuesta de José Álvarez Junco a José Brunner", ivi, pp. 185-188. Ai quali è da aggiungere, per quanto concerne il discusso "pacto del silencio" AGUILAR FERNÁNDEZ Paloma, "Presencia y ausencia de la guerra civil y del franquismo en la democracia española. Reflexiones en torno a la articulación y ruptura del "pacto del silencio"', in ARÓSTEGUI, Julio, GODISCHEAU, François, Guerra civil. Mito y memoria, Madrid, Marcial Pons, 2006, pp. 245-293 e, per una stimolante contestualizzazione, la raccolta di saggi di JULIÁ, Santos, Hoy no es ayer. Ensayos sobre la España del siglo XX, Barcelona, RBA, 2010.

2. PRESTON, Paul, El holocausto español. Odio y exterminio en la guerra civil y después, Barcelona, Debate, 2011.

3. BOLLOTEN, Burnett, The Grand Camouflage: the Communist conspiracy in the Spanish civil war, London, Hollis \& Carter, 1961.

4. ID., La guerra civil española: revolución y contrarrevolución, Madrid, Alianza, 1989.

5. HERNÁNDEZ SÁNCHEZ, Fernando, Guerra o revolución. El Partido Comunista de España en la guerra civil, Barcelona, Crítica, 2010.

6. RANZATO, Gabriele, La grande paura del 1936. Come la Spagna precipitò nella guerra civile, RomaBari, Laterza, 2011, articolazione e approfondimento del precedente ID., L'eclissi della democrazia, Torino, Bollati Boringhieri, 2004. 
7. CRUZ, Rafael, En el nombre del pueblo. República, rebelión militar y guerra en la España de 1936, Madrid, Siglo XXI, 2006.

8. ALÍA MIRANDA, Francisco, Julio de 1936. Conspiración y alzamiento contr ala Segunda República, Barcelona, Crítica, 2011, p. 372.

9. Come continua a ribadire da anni la letteratura apologetica di parte ecclesiastica, di cui l'indiscusso campione è senza ombra di dubbio Vicente Cárcel Ortí, a cui si devono utilissimi repertori di preziose fonti provenienti dagli archivi vaticani, lasciate però orfane di adeguata contestualizzazione e interpretazione, quando non strumentalmente fraintese o piegate a sostegno di una visione confessionale. Cfr. CÁRCEL ORTÍ, Vicente Caídos, Víctimas y martires, Madrid, Espasa, 2008; ID., Pio XI entre la República y Franco, Madrid, BAC, 2008.

10. ANDRÉS-GALLEGO, José, PAZOS RODRÍGUEZ, Antón Mª (eds.), Archivo Gomá. Documentos de la Guerra Civil, CSIC, Madrid, 2001-2010, 13 voll.

11. BOTTI, Alfonso, "Rapporto dell'Azione cattolica sul comunismo in Spagna e uso ecclesiastico del presunto complotto comunista del luglio 1936, alla luce della nuova documentazione vaticana", in Spagna contemporanea, 2010, n. 38, pp. 151-165.

12. BOTTI, Alfonso, "Santa Sede e influenza nazista in Spagna durante la guerra civile nei documenti dell'Archivio Segreto Vaticano", in A. Guasco, R. Perin (eds.), Pio XI: Keywords, ZürichBerlin, LIT Verlag, 2010, pp. 107-129.

\section{AUTORE}

\section{ALFONSO BOTTI}

Alfonso Botti insegna Storia contemporanea presso la Facoltà di Lettere e filosofia dell'Università di Modena-Reggio Emilia.

Negli anni precedenti ha insegnato Storia dei partiti e dei movimenti politici presso la Facoltà di Sociologia dell'Università di Urbino (1991-92); Storia della Spagna contemporanea presso la Facoltà di Lettere e filosofia dell'Università degli studi di Trieste (1992-93 e 1993-94); Lingua, cultura e istituzioni dei paesi di lingua spagnola (1994-95, 1995-96, 1996-97, 1997-98, 1998-99) e Storia della lingua spagnola (1999-2000, 2000-01, 2001-02) presso la Facoltà di Lingue e letterature straniere dell'Università Cattolica del S. Cuore di Milano e Storia contemporanea e Storia dell'Europa presso la Facoltà di Sociologia dell'Università degli Studi “Carlo Bo" di Urbino. Ha usufruito di borse di studio del Fond National de la Recherche Suisse $(1983,1988)$, del Consiglio Nazionale delle Ricerche (1986) e del Ministerio de Asuntos Exteriores spagnolo (1991, 1998). È stato Investigator invitado presso il dipartimento di Storia della Facoltà di Lettere e filosofia dell'Università di Alicante (1994) e ha tenuto un corso di dottorato presso il dipartimento di Storia contemporanea dell'Università di Valencia (1997) e presso l'Università autonoma di Barcellona (2007).

Fa parte di Comitati scientifici del Centro Studi per la storia del modernismo, della Fondazione Romolo Murri entrambi con sede a Urbino, dell'Istituto di studi storici “G. Salvemini” di Torino e del Centre d'Estudis sobre les Èpoques Franquista i Democràtica (CEFID) dell'Università Autonoma di Barcellona.

Fa parte delle redazioni di Fonti e documenti (Urbino), del Comitato di redazione del Bulletin d'Histoire Contemporaine de l'Espagne (Aix-en-Provence), del Comitato di direzione di Storia e 
problemi contemporanei (Ancona) e dei Comitati scientifici delle riviste Studia Histórica. Historia Contemporánea (Università di Salamanca) e Historia del presente (Madrid) dell'Asociación Historiadores del Presente.

Ha collaborato con la RAI Radiotelevisione italiana per i programmi di Telesat e con la casa editrice "il Mulino" per i titoli di storia spagnola contemporanea. Scrive abitualmente sul mensile di recensioni librarie L'Indice e sul bimestrale Il Mulino.

Nel 1997 è risultato vincitore ex-equo nella Sezione cultura marchigiana della XVIª edizione del Premio Nazionale di Cultura Frontino Montefeltro per il volume Romolo Murri e l'anticlericalismo negli anni de "La Voce".

È condirettore della rivista semestrale Spagna contemporanea e dell'omonima collana di studi storici presso le Edizioni dell'Orso di Alessandria.

URL: < http://personale.unimore.it/rubrica/curriculum/ab2761953 > 